\title{
Surface treatment effects on the thickness dependence of the remanent polarization of $\mathrm{PbZr}_{0.52} \mathrm{Ti}_{0.48} \mathrm{O}_{3}$ capacitors
}

\author{
J. Rodríguez Contreras, ${ }^{\text {a) }}$ H. Kohlstedt, U. Poppe, and R. Waser \\ Institut für Festkörperforschung, Forschungszentrum Jülich GmbH, 52425 Jülich, Germany \\ Ch. Buchal \\ Institut für Schichten und Grenzflächen, Forschungszentrum Jülich GmbH, 52425 Jülich, Germany
}

(Received 12 February 2003; accepted 7 May 2003)

\begin{abstract}
In this letter, we report on the thickness dependence of the remanent polarization of $\mathrm{Pt} / \mathrm{PbZr}_{0.52} \mathrm{Ti}_{0.48} \mathrm{O}_{3} / \mathrm{SrRuO}_{3}$ capacitors. Two different patterning techniques were used to fabricate the capacitors. For lift-off processed capacitors, the remanent polarization decreased with decreasing thickness. Ion-beam-etched capacitors, however, showed a constant remanent polarization for all $\mathrm{PbZr}_{0.52} \mathrm{Ti}_{0.48} \mathrm{O}_{3}$ film thicknesses down to $23 \mathrm{~nm}$. Remarkably, this constant remanent polarization for ion-beam-etched capacitors corresponds to the spontaneous polarization expected for a stress-free bulk $\mathrm{PbZr}_{0.52} \mathrm{Ti}_{0.48} \mathrm{O}_{3}$ crystal. (c) 2003 American Institute of Physics.
\end{abstract}

[DOI: $10.1063 / 1.1590431]$

Size effects on ferroelectric (FE) properties have been investigated with a great deal of interest. It is frequently reported that the remanent polarization of a FE material decreases as the film thickness is decreased. In some cases, extrinsic effects, for example, film composition, surface roughness, and defects, are likely to be the reason for this phenomenon. However, given that ferroelectricity is a cooperative phenomenon like superconductivity and magnetism, the size effect is expected to be prominent, even in the absence of extrinsic factors, due to the presence of a film surface. ${ }^{1-4}$ Mechanical and electrical boundary conditions have to be taken into account to calculate the FE film thickness at which the size effect starts playing a role. ${ }^{5-8}$ Our experimental results show that for $\mathrm{Pt} / \mathrm{PbZr}_{0.52} \mathrm{Ti}_{0.48} \mathrm{O}_{3}$ $(\mathrm{PZT}) / \mathrm{SrRuO}_{3}$ (SRO) capacitors deposited on a $\mathrm{SrTiO}_{3}$ (STO) substrate, no size effect is present at a FE film thickness of $23 \mathrm{~nm}$. Therefore, extrinsic effects are likely to be responsible for the suppressed FE properties shown by other groups. ${ }^{9}$ It will be shown in this letter that the surface treatment of the ferroelectric layer is one possible extrinsic effect leading to a decreased remanent polarization.

We have deposited PZT/SRO thin films on STO (100) substrates by high-pressure, on-axis sputtering. ${ }^{10}$ A stoichiometric SRO target was used to grow stoichiometric thin films, whereas a PZT target with a lead excess of $20 \%$ was necessary to compensate the loss of this volatile component. Deposition rates of 12 and $45 \mathrm{~nm} / \mathrm{h}$ were achieved for PZT and SRO, respectively. The optimized substrate temperature and oxygen pressure during growth were about $570^{\circ} \mathrm{C}$ at 3 mbar for both PZT and SRO thin films. Structural characterization techniques, such as $\mathrm{x}$-ray diffractometry, Rutherford backscattering spectrometry, high-resolution transmission electron microscopy, and atomic force microscopy, have

\footnotetext{
a) Author to whom correspondence should be addressed; electronic mail: j.rodriguez@fz-juelich.de
}

shown the excellent crystalline quality of our high-pressure sputtered thin films. ${ }^{11,12}$

To measure FE hysteresis loops, Pt/PZT/SRO heterostructures with PZT thicknesses between 23 and $165 \mathrm{~nm}$ were patterned into capacitors with areas of $25 \times 25$ to 200 $\times 200 \mu \mathrm{m}^{2}$. Two different methods were used to pattern the capacitors. For the first method, photoresist was spun on top of the FE thin film and conventional photolithography defined the area of the capacitors. After sputtering Pt, a lift-off process removed the photoresist together with the $\mathrm{Pt}$ in between the capacitors [Fig. 1(a)]. For the second method, all three layers, Pt/PZT/SRO, were first deposited on the STO substrate. Conventional photolithography and an Ar-etching step, which was timed to stop in the FE layer or at the interface between bottom electrode and FE layer, defined the shape and the area of the capacitors [Fig. 1(b)]. FE hysteresis loops were obtained with the FE module of the aix ACCT TF Analyzer 2000.

FE hysteresis loops have been investigated for both patterning techniques and for all thicknesses. The square shape of the $(P-E)$ curves is softened for thinner films. Imprint is observed for all the loops, but is more pronounced in lift-off processed capacitors and for thinner films. Due to this effect,

(a)
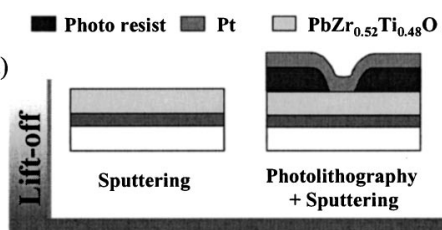

(b)

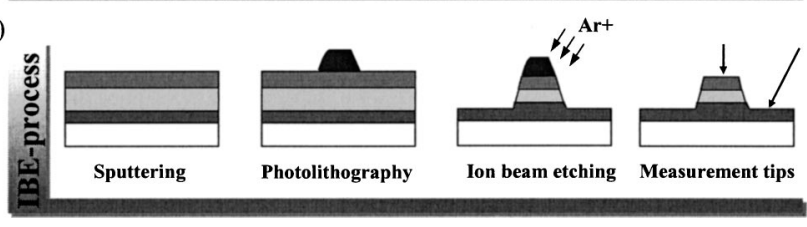

FIG. 1. (a) Patterning of capacitors by lift-off process and (b) patterning of capacitors by ion-beam etching process. 

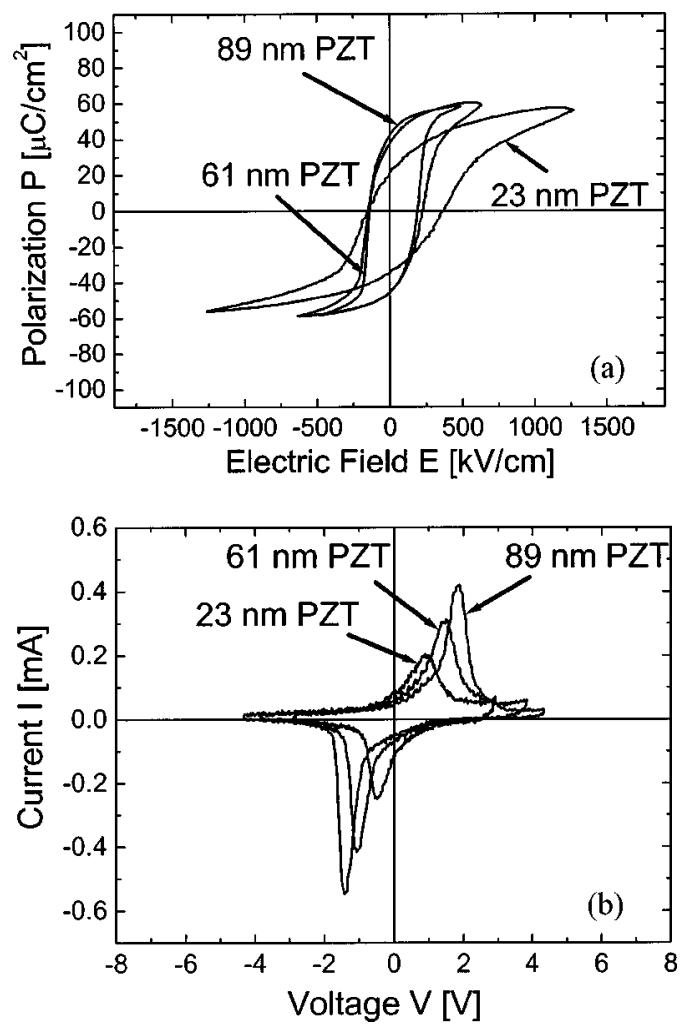

FIG. 2. (a) Hysteresis curves for lift-off processed capacitors and (b) voltage dependence of the current response for lift-off processed capacitors.

the reported values for the remanent polarization and the coercive field are calculated from $\left(P_{r+}+\left|P_{r-}\right|\right) / 2$ and $\left(E_{c+}+\left|E_{c-}\right|\right) / 2$, respectively.

Figures 2(a) and 2(b) show the hysteresis curves and the corresponding voltage dependences of the current response for lift-off patterned PZT capacitors with film thicknesses of $89,61, \mathrm{~nm}$ and $23 \mathrm{~nm}$ measured at $10 \mathrm{kHz}$. The value for the remanent polarization decreases from 44 to $27 \mu \mathrm{C} / \mathrm{cm}^{2}$ as the film thickness is reduced. The coercive field, however, increases for thinner films. It increases from $165 \mathrm{kV} / \mathrm{cm}$ for the 89 -nm-thick film up to $260 \mathrm{kV} / \mathrm{cm}$ for the 23 -nm-thick film.

$P-E$ curves and voltage dependences of the current response for ion-beam etched (IBE) capacitors are shown in Figs. 3(a) and 3(b). The PZT film thicknesses of the shown capacitors are 165 and $23 \mathrm{~nm}$. In this case, no decrease in the remanent polarization is observed. Both capacitors show a remanent polarization of $49 \mu \mathrm{C} / \mathrm{cm}^{2}$. Additional IBE capacitors with PZT film thickness between 165 and $23 \mathrm{~nm}$ have been fabricated and all of them showed similar $P-E$ curves and $P_{r}$ values. The coercive field increases for thinner films. It is as low as $185 \mathrm{kV} / \mathrm{cm}$ for the 165 -nm-thick film and as high as $510 \mathrm{kV} / \mathrm{cm}$ for the 23 -nm-thick film.

The most important result obtained from this work is that for the studied heterostructure, an intrinsic size effect is not observable at a FE film thickness of $23 \mathrm{~nm}$. Each of our IBE capacitors shows the same remanent polarization. We would like to point out that the obtained remanent polarization is the theoretically expected spontaneous polarization for a stress-free bulk PZT crystal of the used composition. ${ }^{13}$ We conclude that either no intrinsic size effect is present in the studied thickness range, or that the intrinsic size effect is Downloaded 21 Dec 2006 to 134.94 .122 .39 . Redistribution subject
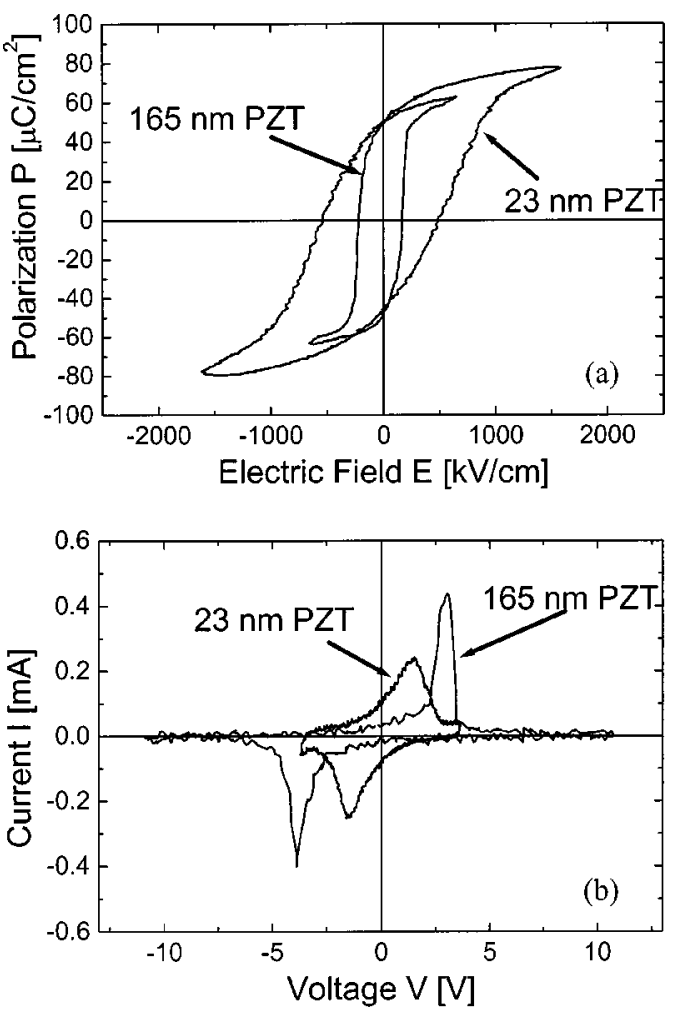

FIG. 3. (a) Hysteresis curves for IBE capacitors and (b) voltage dependence of the current response for IBE capacitors.

compensated by the strain effect. Thin films grown on much thicker substrates are usually strained. As a result, the remanent polarization may increase with decreasing film thickness. This has been predicted theoretically, ${ }^{4}$ as well as observed experimentally. ${ }^{14}$ In fact, our films do show a slight increase of the out-of-plane lattice parameter from 4.132 to $4.140 \AA$ and a decrease of the in-plane lattice parameter from 4.04 to $4.03 \AA$ as the film thickness is reduced from 165 to $23 \mathrm{~nm}$.

The lift-off processed capacitors do not show at any film thickness this theoretically expected value for the remanent polarization. In fact, they show a decreasing $P_{r}$ as the film thickness decreases. We have already ruled out intrinsic effects. In addition, extrinsic effects, such as film composition or film quality, are not suitable to explain this phenomenon, as the thin film deposition was identical for both patterning techniques. However, a strong influence of the interface between the FE films and the electrodes on the switching kinetics seems very likely. The FE film surface is exposed to solvents, photoresist, and developer for the lift-off process. This might lead to an increased surface roughness, resulting in an inhomogeneous field distribution at the PZT-Pt interface. This idea is supported by the reduced coercive field for lift-off processed capacitors compared to IBE capacitors. We believe that the number of nucleation centers may be increased for rougher films, as roughness locally enhances the electric field. As a result, the coercive field is reduced. In addition, the chemical composition of the PZT near an interface could be different, resulting in the formation of interface layers adjacent to the electrode with suppressed FE properties. Whatever occurs to the interface during the lift-off process leads to leakage currents for positive voltages at the top o AIP license or copyright, see http://apl.aip.org/apl/copyright.jsp 
electrode [Fig. 2(b)]. As a consequence of increased leakage currents, the measured remanent polarization is either enhanced or reduced. It is enhanced if, in addition to the displacement current, the leakage current is also integrated. However, it might also be reduced if it is impossible to saturate the $P-E$ curves due to high leakage currents.

We believe that the PZT surface treatment during the lift-off process does result in a reduced remanent polarization for thicker films. In addition, the widely observed increase of the coercive field for thinner films and nonsaturation of the $P-E$ curves due to increased leakage currents at higher voltages are responsible for the decreased remanent polarization for thinner films.

For IBE capacitors, the leakage currents for positive voltages at the top electrode are much lower. Therefore, higher electric fields which are necessary to saturate the $P-E$ curve of capacitors with thinner PZT films can be applied.

We conclude that etch degradation and etching residuals at the edges of the capacitors are less significant than the contact of the PZT surface with chemical solvents during lift-off processing. However, this result might be different for sub-micron-area capacitors for which the influence of the edges is more important.

${ }^{1}$ M. E. Lines and A. M. Glass, Principles and Applications of Ferroelectrics and Related Materials (Oxford University Press, Oxford 1977).

${ }^{2}$ I. P. Batra and B. D. Silverman, Solid State Commun. 11, 291 (1972).

${ }^{3}$ I. P. Batra, P. Würfel, and B. D. Silverman, Phys. Rev. Lett. 30, 384 (1973).

${ }^{4}$ H. Kohlstedt, N. A. Pertsev, and R. Waser, Mater. Res. Soc. Symp. Proc. 688, 161 (2002).

${ }^{5}$ R. Kretschmer and K. Binder, Phys. Rev. B 20, 1065 (1979).

${ }^{6}$ N. A. Pertsev, A. G. Zembilgotov, and A. K. Tagantsev, Phys. Rev. Lett. 80, 1988 (1998).

${ }^{7}$ N. A. Pertsev, A. G. Zembilgotov, and A. K. Tagantsev, Ferroelectrics 223, 79 (1999).

${ }^{8} \mathrm{Ph}$. Ghosez and K. M. Rabe, Appl. Phys. Lett. 76, 2767 (2000).

${ }^{9}$ P. K. Larsen, G. J. M. Dormans, D. J. Taylor, and P. J. van Veldhoven, J. Appl. Phys. 76, 2405 (1994).

${ }^{10}$ U. Poppe, J. Schubert, R. Arons, W. Evers, C. H. Freiburg, W. Reichert, K. Schmidt, W. Sybertz, and K. Urban, Solid State Commun. 66, 661 (1988).

${ }^{11}$ J. Rodríguez Contreras, J. Schubert, U. Poppe, O. Trithaveesak, K. Szot, Ch. Buchal, H. Kohlstedt, and R. Waser, Mater. Res. Soc. Symp. Proc. 688, C8.10 (2002).

${ }^{12}$ C. L. Jia, J. Rodríguez Contreras, U. Poppe, H. Kohlstedt, R. Waser, and K. Urban, J. Appl. Phys. 92, 101 (2002).

${ }^{13}$ M. J. Haun, E. Fuhrman, S. J. Jang, and L. E. Cross, Ferroelectrics 99, 63 (1989).

${ }^{14}$ N. Yanase, K. Abe, N. Fukushima, and T. Kawakubo, Jpn. J. Appl. Phys. 38, 5305 (1999). 